\title{
C3: connect separate connected components to form a succinct disease module
}

\author{
Bingbo Wang ${ }^{1 *} \mathbb{0}$, Jie Hu', Yajun Wang ${ }^{2}$, Chenxing Zhang ${ }^{1}$, Yuanjun Zhou', Liang Yu ${ }^{1}$, Xingli Guo ${ }^{1}$, Lin Gao ${ }^{1}$ \\ and Yunru Chen ${ }^{3 *}$
}

*Correspondence: bingbowang@xidian.edu. cn; chenyunru_2002@xjtu. edu.cn

${ }^{1}$ School of Computer Science and Technology, Xidian University, Xi'an, People's Republic of China ${ }^{3}$ The First Affiliated Hospital of Xi'an Jiaotong University, Xi'an, People's Republic of China

Full list of author information is available at the end of the article

\begin{abstract}
Background: Precise disease module is conducive to understanding the molecular mechanism of disease causation and identifying drug targets. However, due to the fragmentization of disease module in incomplete human interactome, how to determine connectivity pattern and detect a complete neighbourhood of disease based on this is still an open question.
\end{abstract}

Results: In this paper, we perform exploratory analysis leading to an important observation that through a few intermediate nodes, most separate connected components formed by disease-associated proteins can be effectively connected and eventually form a complete disease module. And based on the topological properties of these intermediate nodes, we propose a connect separate connected components (C3) method to detect a succinct disease module by introducing a relatively small number of intermediate nodes, which allows us to obtain more pure disease module than other methods. Then we apply C 3 across a large corpus of diseases to validate this connectivity pattern of disease module. Furthermore, the connectivity of the perturbed genes in multi-omics data such as The Cancer Genome Atlas also fits this pattern.

Conclusions: C3 tool is not only useful in detecting a clearly-defined connected disease neighbourhood of 299 diseases and cancer with multi-omics data, but also helpful in better understanding the interconnection of phenotypically related genes in different omics data and studying complex pathological processes.

Keywords: Disease module, Connectivity pattern, Biological network

\section{Background}

Most complex human diseases are rarely the consequence of the abnormal activity of a single gene product. Conversely, they involve the activities of multiple interacting genes, yet many of which are not defective [1]. Hence, it is crucial to study the relationship between genotypes and phenotypes to unlock the genetic origins of complex diseases [2-6] in the context of human interactome (molecular interactions networks, nodes are proteins and edges are observation and inference from their interactions). Driven by high-throughput interactome mapping efforts [7] and the genome-wide genetic association data [8], previous work has begun to unveil the relationships between different author(s) and the source, provide a link to the Creative Commons licence, and indicate if changes were made. The images or other third party material in this article are included in the article's Creative Commons licence, unless indicated otherwise in a credit line to the material. If material is not included in the article's Creative Commons licence and your intended use is not permitted by statutory regulation or exceeds the permitted use, you will need to obtain permission directly from the copyright holder. To view a copy of this licence, visit http:// creativecommons.org/licenses/by/4.0/. The Creative Commons Public Domain Dedication waiver (http://creativecommons.org/publi cdomain/zero/1.0/) applies to the data made available in this article, unless otherwise stated in a credit line to the data. 
phenotypes based on network. The most important concept that emerges from this is the "disease module". Disease-associated proteins, (DAPs, encoded by disease genes) for a specific disease phenotype show a tendency to cluster in the same network neighbourhood [3]. Topological properties of disease module in the interactome underlie all network-based prioritization tools for studying human diseases. Topological characteristics of existing studies include: specific phenotype-related DAPs frequently form a well-connected component rather than locally dense communities [9]; most disease genes show no tendency to encode hub proteins, but rather are located in the functional periphery of interactome [1]; furthermore, the position of each disease module relative to other modules in the interactome implies its phenotypic similarity to other diseases [10]; and recently, Agrawal et al. [11] have found that DAPs of a single disease tend to form many separate connected components in the network. Their research also shows that higherorder network structures such as small subgraphs of the pathway can provide a promising direction for the development of new methods.

Disease module helps to uncover the molecular mechanism of disease causation, identify new disease genes and pathways and drug targets [9, 12-18]. For example, Sharma et al. [18] have identified the disease module of asthma and verified its functional and pathophysiological correlation using both computational and experimental approaches. They found that the asthma disease module had a modest GWAS $p$ values against the background of random variation, and there are differentially expressed genes in normal and asthmatic fibroblast cells treated with an asthma-specific drug. The asthma module also contains immune response mechanisms that are shared by other immune-related disease modules.

Numerous computational methods have been developed for the accurate identification of disease module to fully explain the molecular mechanism of most human diseases. With regard to the fragmentation of disease module [10], more than $80 \%$ of DAPs on average are shown to be scattered, forming many separate connected components (SCCs), this pattern is applicable to more than $90 \%$ diseases [19]. A disease module does not correspond to a single well-connected component as an observable module in present incomplete interactome. Therefore, many methods $[9,14,20,21]$ used for searching for DAPs-intensive clusters or communities within the interactome might have limitations in discovering disease modules. Such as DIAMOnD [9], the most advanced algorithm, is used to analyze the connectivity patterns of DAPs and form a disease module with a large number of intermediate proteins, which are known as DIAMOnD proteins. Although DIAMOnD can generate internal interconnected module for a specific disease, unfavourably, on average it introduces 200 DIAMOnD proteins, but only connects about average 50\% DAPs of 299 diseases from Online Mendelian Inheritance in Man (OMIM [22]) and Genome-Wide Association Studies (GWAS [8]) databases. Many of the introduced DIAMOnD proteins are unnecessary for precisely detecting disease modules, resulting in enormous disease module sizes (average size of 400 proteins, i.e. the disease module has been magnified 4 times), ultimately the analysis of 299 disease relationships based on such modules is ambiguous. Another algorithm is based on the Steiner tree, the retrieval of a minimum size sub-graph leads to the classic Steiner tree problem, which is known as NP-complete [23], such as a heuristic method based on the minimum spanning tree approximation, the time complexity of the entire algorithm is 
about $\mathrm{O}\left(\mathrm{n}^{3}\right)$. This is impractical to detect disease modules in a large human interactome of more than 13,000 proteins, as it is a time-consuming process of finding the optimal connected components. On the other hand, any remote nodes can be connected through a Steiner tree, the connection properties of the disease module are therefore hindered by these problems, which may lead to the misunderstanding of the topological characteristics of DAPs and disease modules. Overall, despite many methods, the analysis of connectivity pattern of DAPs are still an unsolved problem.

In this paper we proposed a topological-based method to connect separate connected components $(\mathrm{CCC}, \mathrm{C} 3)$ in order to form a succinct disease module. We showed that SCCs formed by DAPs can be effectively connected through a few intermediate proteins and interactions in the network, which can statistically significantly reduce the number of fragments of a disease module. The specific process is as follows. First, we quantified the roles of proteins and interactions in connecting SCCs, i.e. by importing the intermediate proteins and interactions, C3 detects observable disease modules for DAPs of 299 disease phenotypes from the OMIM [22] and GWAS [8] databases. Next, we described the characteristics of the detected disease modules, we verified the succinctness and connectivity significance of $\mathrm{C} 3$ module by comparing this method with DIAMOnD and random simulations results. C3 module is a well-connected succinct disease module with small-size and dominated by DAPs. Additionally, we explored their relative contributions to disease pathogenesis in some case studies. And we presented evidence suggesting that even for DAPs from multi-omics data in The Cancer Genome Atlas (TCGA [24]), C3 worked as a practical strategy for understanding interrelationships between mRNA expression, mutation, DNA methylation and copy number variation, which further validated the biological significance of the disease module we obtained.

\section{Results}

\section{Connectivity pattern of disease module}

To explore the connectivity pattern of disease module, we described our framework (Fig. 1a) as follows: (1) the human interactome and the Disease-Associated Proteins (DAPs) data for model building, (2) detection of Separate Connected Components (SCCs) forming by DAPs in the interactome, (3) quantification index of the connectivity ability of intermediate proteins, (4) greedy process of C3 algorithm, and (5) quantification of succinctness of final disease module.

We detected the connectivity pattern of disease module in a comprehensive list of experimental records of molecular interactions in human cells compiled by Menche et al. [10] (see Methods). In total, we obtained 141,296 interactions among 13,460 proteins. This human interactome can be modeled as an undirected network $G(N, E)$, where $N$ is the proteins set and $E$ is the interactions set. The known DAPs of 299 diseases are gathered from OMIM [22] and GWAS [8] databases (see Methods), of which 2436 are in $N$ and associated with one or more diseases. We call these 2436 DAPs "seeds" in our subsequent algorithm process.

The SCCs forming by DAPs in $G$ are identified by a tool networkx [25] in python which can export all the connected components of a given node set. We used a function ExScc (see Methods) to export the set of SCCs of a given seed set in our method. Based on this, 


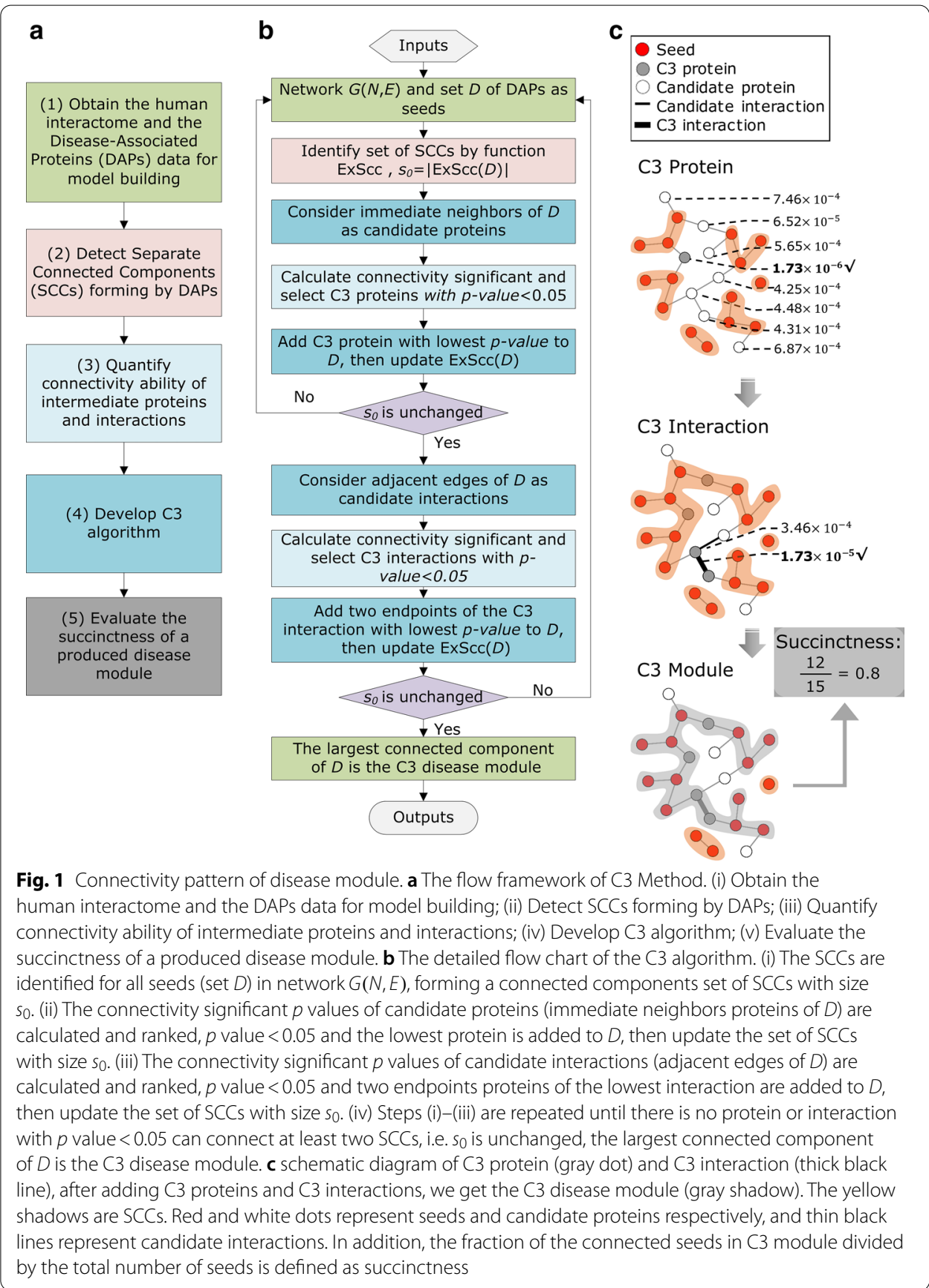

the connectivity patterns of 299 typical complex diseases in a curated list are studied (see results in Additional file 1).

To validate whether there are some intermediate proteins or interactions can statistically significantly reduce the number of fragments of a disease module or not, we used hypergeometric $p$ value to quantify their ability in connecting SCCs of DAPs. The $p$ value evaluates whether a certain intermediate node or edge can connect more SCCs than expected (see Methods). We found that there are certainly some intermediates can significantly connect SCCs and reduced the number of them ( $p$ value $<0.05$, connectivity 
is significant). A "C3 protein" or a "C3 interaction" is defined as a node or an edge with $p$ value $<0.05$ and lowest (Fig. 1b, c). The $\mathrm{C} 3$ proteins and the pair of proteins occupying the endpoint of C3 interactions are collectively referred to as "C3 proteins" in our functional analysis below. Then we formulated the disease module identification problem as a C3 proteins detection problem: Succinct disease module detection problem. Given a network $G(N, E)$ and a seed set $D, \operatorname{ExScc}(D)$ is a function which can export the set of SCCs of $D$, find a node set $C$ with minimum size, such that $\forall c \in C, c$ is a $C 3$ protein, and $\min _{C}|\operatorname{ExScc}(D \cup C)|$. The initial seed set $D$ forms lots of SCCs, and presents a large $|\operatorname{ExScc}(D)|$, the size of SCCs will significantly decrease as introducing $C 3$ protein set $C$ into disease module. A small $|\operatorname{ExScc}(D \cup C)|$ means that there are $\mathrm{C} 3$ proteins can significantly reduce the number of fragments of a disease module. Extremely $|\operatorname{ExScc}(D \cup C)|=1$ indicates that all the DAPs can be connected by C3 proteins into one component in the network. Then we developed a greedy process (C3 algorithm in Fig. 1b, see Methods for details) to efficiently solve this problem, by importing C3 proteins and C3 interactions iteratively, and eventually a well-connected disease module becomes observable (Fig. 1c). Specifically the largest connected component (LCC) of $D \cup C$ are named $C 3$ module.

Furthermore, previous methods such as DIAMOnD [9], also construct a disease module by introducing some intermediate proteins. Disease Module is the LCC of a union set of DAPs and intermediate proteins. It is obvious that more DAPs are connected by intermediate proteins, the final disease module are more dominated by known DAPs. Therefore, we proposed the fraction of connnected DAPs in the disease module $(|\mathrm{D} \cap \mathrm{Dm}| /|\mathrm{D}|)$ as an index to quantify the succinctness of a produced disease module $D m$ (Fig. 1c). The succinctness can be used to compare the purity between results of different methods. It is a value between 0 and 1 , and the closer to 1 , the more succinct the disease module is.

\section{Succinctness and connectivity significance of the $\mathrm{C} 3$ module}

According to disease module hypothesis, genes associated with a specific disease phenotype tend to cluster in the same network neighborhood, usually referred to as a "disease module". Furthermore, the location of each disease module relative to others in the network implies its phenotypic similarity to other diseases [10], and the position of the disease module in the network is of great importance. Through a basic easy-to-understand detection process, we could obtain $\mathrm{C} 3$ disease module, $\mathrm{C} 3$ proteins and $\mathrm{C} 3$ interactions (Fig. 1c) for a particular disease (see Methods). In DIAMOnD disease module, due to the large number of the DIAMOnD proteins, DIAMOnD module is a huge one dominated by DIAMOnD proteins, it would bring many false signals to the disease module. Comparatively, C3 disease module imports a small amount of $\mathrm{C} 3$ proteins and is dominated by DAPs, the $\mathrm{C} 3$ disease module can accurately pinpoint the area affected by the disease on the network and provide us with more accurate disease signals. Therefore, a succinct disease module is essential for studying disease occurrence and treatment.

As mentioned above, the 299 disease modules obtained by the DIAMOnD algorithm are redundant. We compared the $\mathrm{C} 3$ to the DIAMOnD algorithm on a data platform that is more conducive to DIAMOnD using the DIAMOnD supporting data, which includes: human interactome network and the curated list of 299 diseases (see Methods). 
We found that in human interactome C3 can effectively connect more seeds with a $p$ value of $3.94 \mathrm{e}-99$ (wilcoxon rank sum test) and its succinctness is about $95 \%$ while the succinctness of DIAMOnD is about $39 \%$ by using the same intermediate proteins. In addition, we used different types of systematic networks BioGRID, Bioplex and HuRI. Network information is shown in Table 1. In different types of networks, when the same intermediate proteins are imported, C3 can more effectively connect more seeds than DIAMOnD, and the differences in $p$ values of succinctness are respectively $5.80 \mathrm{e}-98$, 4.09e-96, 1.58e-57 (Fig. 2a). This fully demonstrates the succinctness of the C3 module.

Then we chose asthma and BReast CAncer (BRCA) as examples for detailed analysis (Fig. 2b-g). For asthma, the same 86 asthma seeds are connected by the DIAMOnD and C3, C3 proteins require 27 intermediate proteins while DIAMOnD uses 350 intermediate proteins, which is about 13 times that of C3. The same is for BRCA. To connect 29 BRCA seeds, 7 C3 proteins and 350 DIAMOnD proteins are required. The number of the imported DIAMOnD proteins is about 50 times that of C3 (Fig. 2b, e). Therefore, using fewer $\mathrm{C} 3$ proteins can connect the same number of the seeds, further proving the advantages of C3 method in connecting seeds. C3 method is equally applicable to other diseases (Fig. 2a)

Connectivity significance refers to the ubiquitousness of $\mathrm{C} 3$ proteins and $\mathrm{C} 3$ interactions in the network. They can significantly connect SCCs of seeds, i.e., they can play a role in the connection of disease modules. To test whether the observed disease modules represent non-random aggregation of DAPs, we performed randomized experiments on asthma and BRCA (see Methods). For asthma, we found that completely random modules of the same size (add 100 intermediate proteins as DIAMOnD) contained an average of $44.9 \pm 3.2$ seeds, which was significantly lower than the number of seeds in the real disease module, both DIAMOnD module (contains 69 seeds, z-score $=8.6$ ) and C3 module (contains 111 seeds with only $61 \mathrm{C} 3$ proteins, $\mathrm{z}$-score $=21.6$ ) (Fig. $2 \mathrm{c}$ ), even if the proteins are randomly chosen only from the immediate neighbors of seeds, it is observed that the resulting modules contain only $60.3 \pm 4.7$ seeds, which is again significantly lower compared with the DIAMOnD module $(\mathrm{z}$-score $=1.8)$ and $\mathrm{C} 3$ module $(\mathrm{z}$-score $=10.7)($ Fig. $2 \mathrm{~d})$. For the 40 DAPs of BRCA, we randomly selected 40 proteins with the same seed degree for random experiments. We used $35.6 \pm 4.4$ intermediate proteins to connect $38.7 \pm 1.6$ seeds which were randomly selected. It is shown that C3 not only connects all 40 seeds $(\mathrm{z}$-score $=0.8)$ and uses fewer intermediate proteins $(\mathrm{z}$-score $=-2.6)$ compared

Table 1 Network information

\begin{tabular}{|c|c|c|c|c|c|}
\hline Network & Source & $\begin{array}{l}\text { \#Nodes } \\
\text { \#Edges }\end{array}$ & Mean degree & Diameter & $\begin{array}{l}\text { Clustering } \\
\text { coefficient }\end{array}$ \\
\hline Human Interactome & Menche et al. [10] & $\begin{array}{l}13,460 \\
141,296\end{array}$ & 20.6 & 13 & 0.17 \\
\hline BioGRID & https://thebiogrid.org/ & $\begin{array}{l}17,741 \\
338,793\end{array}$ & 37.9 & 8 & 0.12 \\
\hline Bioplex & https://bioplex.hms.harvard.edu/ & $\begin{array}{l}10,961 \\
56,553\end{array}$ & 10.3 & 11 & 0.09 \\
\hline HuRl & $\begin{array}{l}\text { https://interactome.dfci.harva } \\
\text { rd.edu/H_sapiens/ }\end{array}$ & $\begin{array}{l}7,352 \\
38,414\end{array}$ & 10.3 & 11 & 0.06 \\
\hline
\end{tabular}



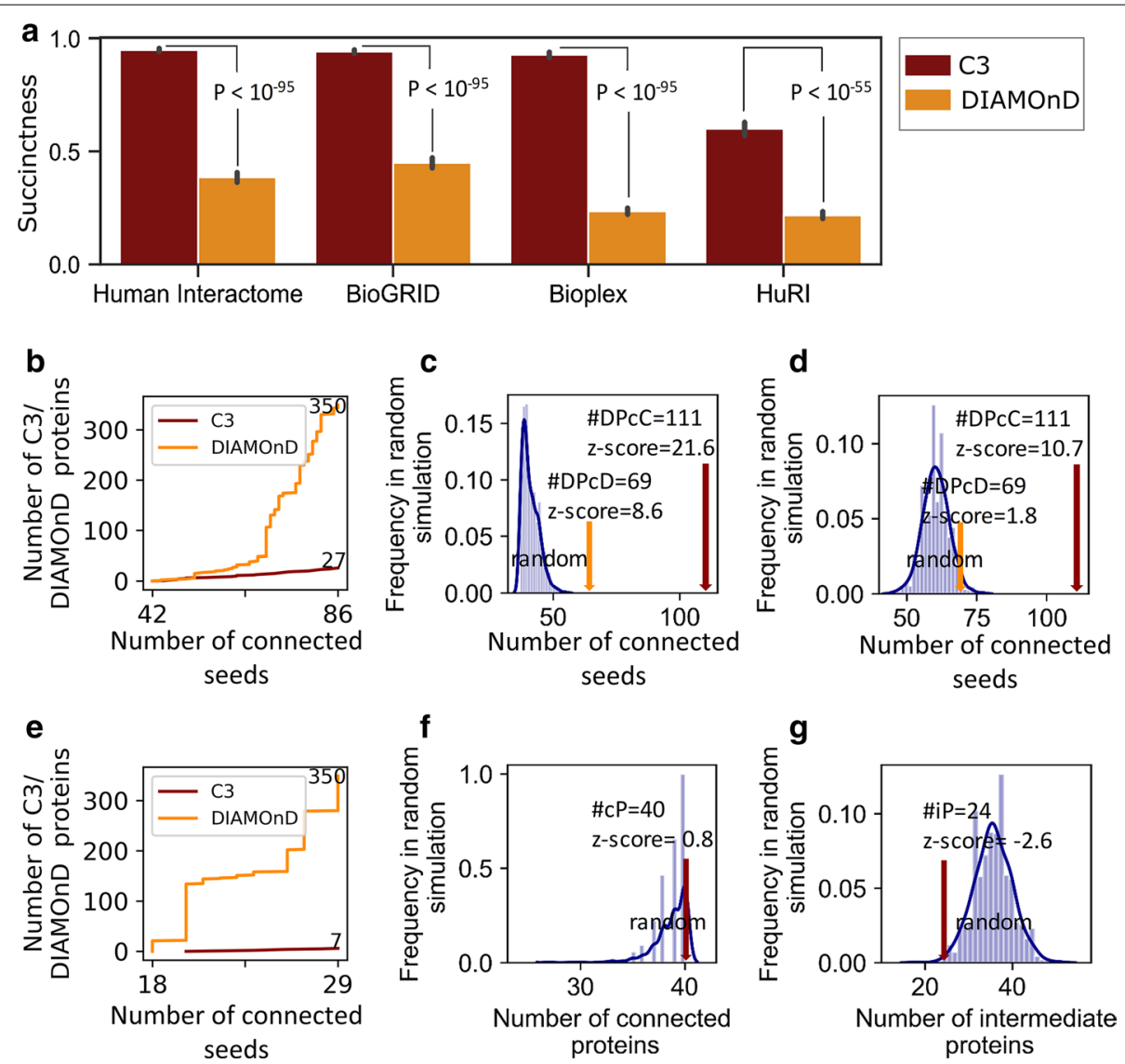

Fig. 2 Succinctness and connectivity significance of the C3 module. a The succinctness of the C 3 and DIAMOnD modules of 299 diseases in different networks, and $p$ value is obtained by wilcoxon rank sum test. b-d For asthma, $\mathbf{b}$ when the modules obtained by DIAMOnD and C3 contain 86 seeds at the same time, red line indicates that only 27 C3 proteins are used in the C3 module, while yellow line indicates that 350 DIAMOnD proteins are used in the DIAMOnD module. c Comparison of the number of Disease Proteins contained in DIAMOnD module (DPCD) and C3 module (DPCC) with 1000 random simulations. Completely randomly-constructed modules contain an average of $44.9 \pm 3.2$ seeds, which is significantly lower than the value in the real disease module. And DIAMOnD module containing 69 seeds ( $z$-score $=8.6$ ) is lower than C3 module with 111 seeds ( $z$-score $=21.6)$. $\mathbf{d}$ Similar to that in $(\mathbf{c})$, but intermediate proteins are not chosen completely at random, but only from the immediate neighbor of seeds. The random modules contain $60.3 \pm 4.7$ seeds, which is again significantly lower than in the real disease module. And DIAMOnD module containing 69 seeds ( $z$-score $=1.8$ ) is also lower than C3 module with 111 seeds ( $z$-score $=10.7$ ). $\mathbf{e}-\mathbf{g}$ With BRCA there are 40 DAPs (seeds), e Same as B, when DIAMOnD and C3 module contain 29 seeds at the same time, only 7 C3 proteins are used in the C3 module while 350 DIAMOnD proteins are used in the DIAMOnD module. Comparison of the number of connected Proteins ( $\mathrm{CP}$ ) (f) and the number of intermediate Proteins (iP) (g) in the final C3 module with the number obtained from 1000 random simulations. Randomly 40 proteins are selected with the same seed degrees each time. The average number of connected seeds is $38.7 \pm 1.6$, while C 3 connected all 40 BRCA proteins. The number of intermediate proteins used on average is $35.6 \pm 4.4$, and only 24 C3 proteins are used in BRCA C3 module

to random simulations (Fig. 2f, g). These differences successfully demonstrate the connectivity significance of the disease module in the interactome, indicating the significant connecting tendency of DAPs. And the C3 disease module is superior to DIAMOnD in revealing the connection patterns of DAPs in the interactome. 


\section{C3 module in asthma}

We have obtained C3 modules of 299 diseases. Can these modules effectively characterize the corresponding diseases? In the following, we analyzed asthma C3 module and compared it with DIAMOnD module. The asthma test mentioned above is based on 139 DAPs from five sources: (1) Literature; (2) GWAS; (3) OMIM; (4) MeSH; (5) Pathways [18], 129 (92.8\%) of which are in the human interactome network. Then we projected them onto the interactome and obtained the SCCs (Fig. 3a). It was observed that the seeds formed a large number of SCCs ( 83 SCCs) in the real interactome. Then, $\mathrm{C} 3$ method was used to connect these SCCs to obtain a well-connected module of asthma. We used 61 C3 proteins to well connect 65 (78\%) SCCs, inferring that the SCCs tend to be connected rather than dispersed (Fig. 3b). In addition, we need biological enrichment analysis to verify the significance of this asthma C3 module. For unconfirmed C3 proteins in this module, its relationship with asthma has not yet been found, and we have suggested this possibility through enrichment analysis and provided clues for the diagnosis and treatment of asthma. We used three different asthma-specific validation data: (1) Gene expression of asthma-specific compiled from eight sources; (2) Gene Ontology (GO) term similarity (biological processes, BP); (3) Disgenet (see Methods and Fig. 3c). The similarity of GO term is calculated by using the tool GOSemSim [26]. In these three assessments, we observed that enrichment of $\mathrm{C} 3$ and DIAMOnD proteins can reach the same level. And the level of enrichment $p$ value of C3 is comparable to DIAMOnD and even better sometimes. These three enrichment analyses show that $\mathrm{C} 3$ proteins have biological significance, and it is speculated that it is biologically related to asthma, so the $61 \mathrm{C} 3$ proteins and the connected seeds form C3 module, which is a reliable asthma module.

Furthermore, in order to better dig into the role of $\mathrm{C} 3$ proteins in the underlying disease pathways, we used DAVID [27] (https://david.ncifcrf.gov/) to study the functional annotation of the $61 \mathrm{C} 3$ proteins in asthma (Fig. 3d). Here we focused on the function annotations of GO terms (BP) and KEGG pathway. We found that C3 proteins enrichment in some pathways has a lower $p$ value, which has been reported to be associated with asthma (PMID), such as: neuropeptide Y may promote TH2 inflammatory response in asthma [28], signal transduction of IL-13 plays a role in the pathogenesis of bronchial asthma [29], humoral immune responses during asthma [30]. And in Table 2, we present in detail the enrichment function and enriched C3 genes

\footnotetext{
(See figure on next page.)

Fig. 3 The visualization and biological explanation of C3 module for asthma. a Subgraph of the full interactome showing the connections among the asthma DAPs. Asthma DAPs are scattered within 83 SCCs in the interactome (shown in shades). The colors of the nodes indicate the source of each seed. There are five sources: (i) Literature; (ii) GWAS; (iii) OMIM; (iv) MeSH; (v) Pathways. b C3 module of Asthma. There are 111 seeds (red nodes) and 61 C3 proteins (grey nodes) in the module. The interaction between them is also indicated in the module. $\mathbf{c}$ Biological interpretation of C3 module for asthma. We consider three verification datasets: (i) Gene expression of asthma-specific compiled from eight sources; (ii) GO term similarity (BP); (iii) Disgenet. Enrichment shows the fraction of C3 (Deep red line), DIAMOnD (green line), seeds (short dotted red line) and random (Dotted blue line) proteins in different validation datasets, statistical significance shows the corresponding statistical test $p$ values, which are derived from the hypergeometric. (D) Functional annotation of GO term (BP) and KEGG pathway ( $p$ value $<0.01$ ) of C3 proteins using DAVID. The pink bar indicates the $-\log _{10}$ operation on the enriched $p$ value
} 


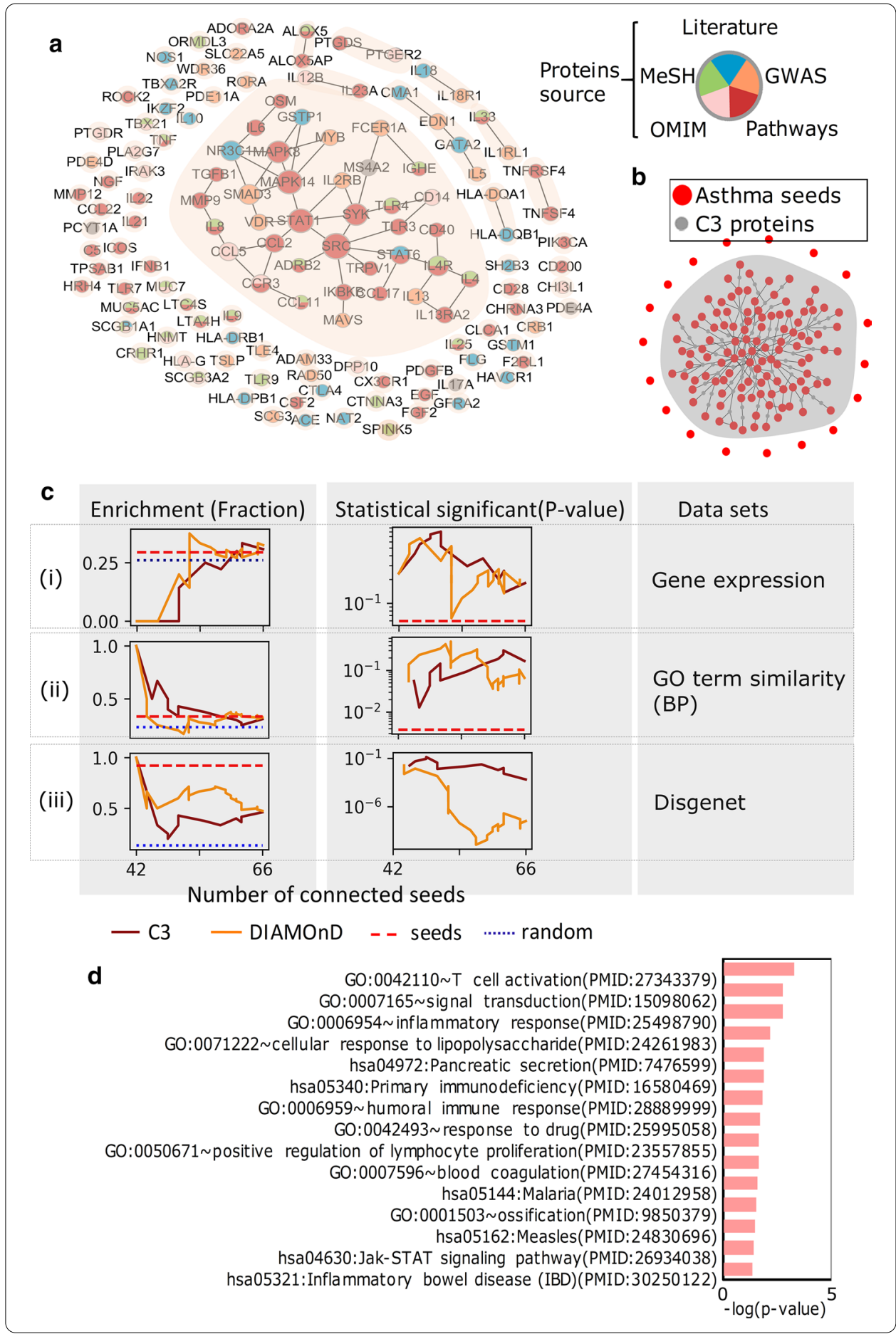

as well as the PMID verified by the literature. Some C3 proteins are highly associated with asthma, such as: CD80 influences synthesis of IL-4 and IFN in non-specific bronchial asthma [31], mutations in IL12A influence cockroach allergy among children with asthma [32], ADA polymorphisms are related to asthma [33]. It indicates 
Table 2 GO term and KEGG pathway functional annotation of C3 proteins and literaturevalidated PMID of C3 genes associated with asthma

\begin{tabular}{|c|c|c|}
\hline GO term/KEGG & \#Genes & Gene(PMID) \\
\hline GO:0042110 & 4 & CD80(PMID:14692664), ADA(PMID:16754522), FOXP3(PMID:26646898) \\
\hline GO:0007165 & 12 & $\begin{array}{l}\text { CRH(PMID:10423904), ERBB3(PMID:1 1799369), IL9R(PMID:1 1039580), } \\
\text { MYD88(PMID:27289030), PTGES(PMID:27611488) }\end{array}$ \\
\hline GO:0006954 & 7 & $\begin{array}{l}\text { CCR4(PMID:291 18379), CRH(PMID:10423904), IL37(PMID:29137646), } \\
\text { MYD88(PMID:27289030) }\end{array}$ \\
\hline GO:0071222 & 4 & $\begin{array}{l}\text { CD80(PMID:14692664), B2M(PMID:26051416), IL12A(PMID:18671862), } \\
\text { TFPI(PMID:16095153) }\end{array}$ \\
\hline hsa05340 & 3 & ADA(PMID:16754522) \\
\hline GO:0006959 & 3 & EBI3(PMID:28553015), LTF(PMID:22837640) \\
\hline GO:0042493 & 5 & ADA(PMID:16754522), B2M(PMID:26051416), CRH(PMID:10423904) \\
\hline GO:0050671 & 2 & IL12A(PMID:18671862), MYD88(PMID:27289030) \\
\hline GO:0007596 & 4 & PRTN3(PMID:29665127), TFPI(PMID:16095153) \\
\hline hsa05144 & 3 & IL12A(PMID:18671862), MYD88(PMID:27289030) \\
\hline GO:0001503 & 3 & SP3(PMID:23057572), LTF(PMID:22837640) \\
\hline hsa05162 & 4 & IL12A(PMID:18671862), MYD88(PMID:27289030) \\
\hline hsa04630 & 4 & IL12A(PMID:18671862), IL9R(PMID:1 1039580) \\
\hline
\end{tabular}

that the C3 module has biological significance. To be specific, it can explain the disease mechanism and help predict potential pathogenic genes or novel drug targets.

\section{C3 module in TCGA multi-omics data of BRCA}

C3 method can be used not only for DAPs, but also for the analysis of genome-wide to obtain genome-wide [16] disease neighborhoods. We collected four omics data: (1) mRNA expression; (2) mutation; (3) DNA methylation from TCGA and (4) copy number variation (see Methods), and successfully identified multi-omics neighborhoods of BRCA. In order to discover the connections between multi-omics data, we used the same human interactome network. For multi-omics data of BRCA, we identified the mutated genes and imported the encoded proteins as seeds into $\mathrm{C} 3$ method to obtain their respective $\mathrm{C} 3$ modules (Fig. 4a). Then, at different omics levels, we compared mRNA expression, mutation, DNA methylation of $\mathrm{C} 3$ proteins. We found that the $\mathrm{C} 3$ proteins imported from different omics data have definite omics specificity. For example, in mRNA expression omics, the mRNA expression of the imported C3 proteins is highly disturbed while its mutation and DNA methylation are not disturbed to a high degree. But the only thing that is special is for the C3 proteins imported by copy number variation, their mRNA expression and mutation levels are also high (Fig. 4b).

For the same disease, is there any potential association between four omics data? We further want to see the relationship of their respective C3 proteins, we used the overlap to represent. As a result, we found that there is no significant association between four omics data, although all are related to the same disease. The same for their respective $\mathrm{C} 3$ proteins, there is no overlap between them, which indicates that C3 proteins of the four omics data are also specific. That is, for BRCA, four specific omics data correspond to four specific groups of C3 proteins (Fig. 4c). 

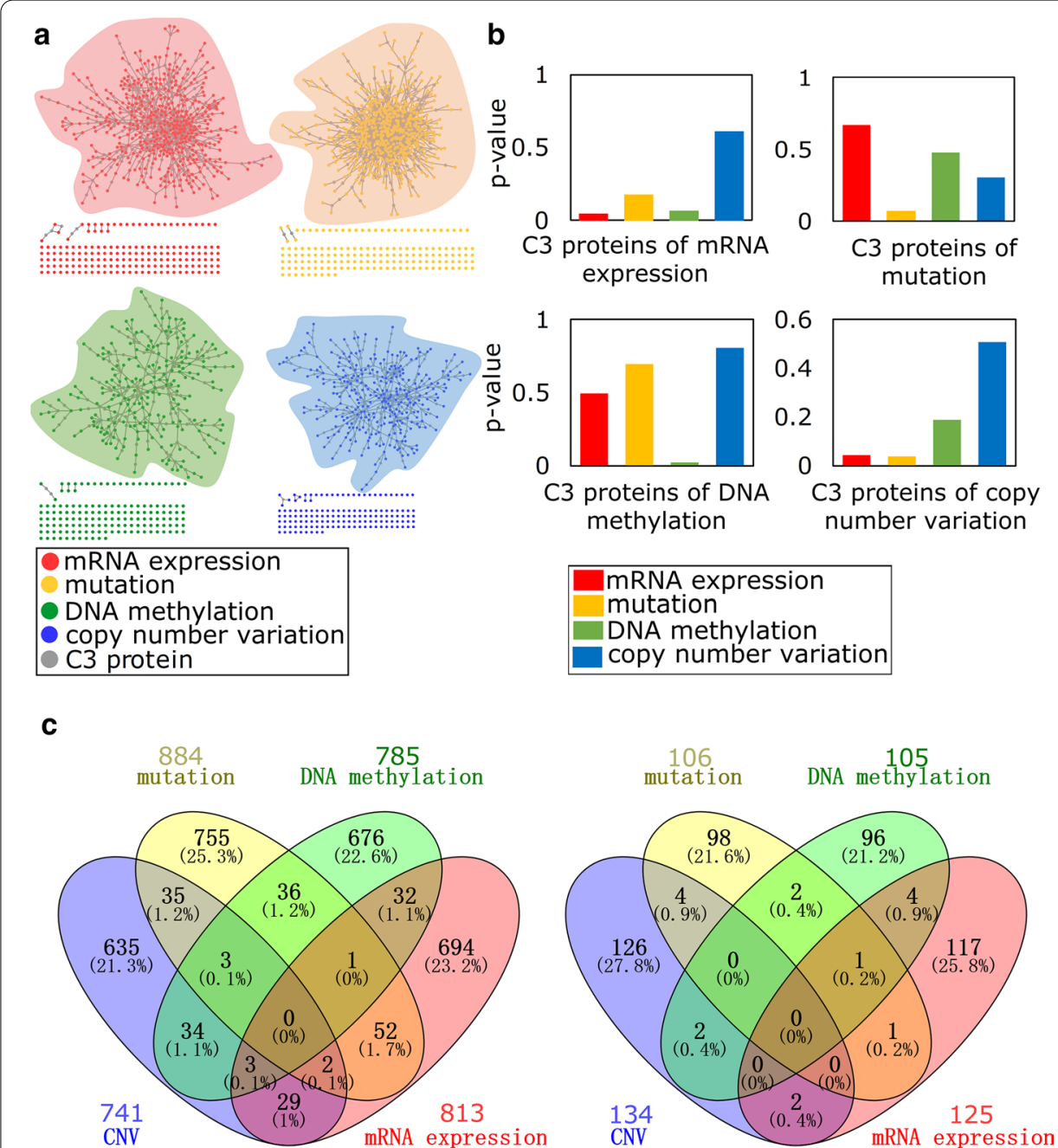

Fig. 4 Specificity of C3 module in TCGA multi-omics data of BRCA. a The C3 module of four omics data of BRCA: (i) mRNA expression; (ii) mutation; (iii) DNA methylation; (iv) copy number variation. b Specificity of C3 proteins imported by different omics data. Red, yellow, green, and blue bars indicate the degree of perturbation of the $\mathrm{C} 3$ proteins imported by the four omics data, compared to the rest of the network ( $p$ value obtained by Wilcoxon test). $\mathbf{c}$ Venn diagram of different omics data (left) and four different C3 proteins we obtained (right)

Table 3 Biological significance of four omics of C3 proteins of BRCA

\begin{tabular}{lllll}
\hline Data (\#proteins) & $\begin{array}{l}\text { mRNA expression } \\
(\mathbf{1 2 5}) \\
\text { \#proteins }(\boldsymbol{p} \text { value) }\end{array}$ & $\begin{array}{l}\text { Mutation(106) } \\
\text { \#proteins } \\
(\boldsymbol{p} \text { value) }\end{array}$ & $\begin{array}{l}\text { DNA methylation } \\
\mathbf{( 1 0 5 )} \\
\text { \#proteins ( } \boldsymbol{p} \text { value) }\end{array}$ & $\begin{array}{l}\text { CNV(134) } \\
\text { \#proteins ( } \boldsymbol{p} \text { value) }\end{array}$ \\
\hline OMIM(7952) & $100(0)$ & $75(0.0056)$ & $85(0)$ & $91(0.01652)$ \\
GWAS(5022) & $61(0.00371)$ & $57(0.00022)$ & $50(0.01305)$ & $53(0.2789)$ \\
Drug-target(2635) & $40(0.00035)$ & $32(0.00328)$ & $38(0.00002)$ & $34(0.04129)$ \\
\hline
\end{tabular}

PPI network: 13,460 proteins. The italics number means less than 0.05

In Table 3, we validated biological significance of four groups of omics of C3 proteins of BRCA, we considered three verification datasets: (1) OMIM; (2) GWAS; (3) Drugtarget, for each validation dataset, we used the Wilcoxon test for statistical significance 
analysis of enrichment. OMIM and GWAS databases can indicate the relationship between $\mathrm{C} 3$ proteins and diseases, and Drug-target database can show the relationship between $\mathrm{C} 3$ proteins and the drug target. We find that the $\mathrm{C} 3$ proteins corresponding to these four omics data have different degrees of enrichment significance in these three verification datasets, which indicates that the $\mathrm{C} 3$ proteins identified by our $\mathrm{C} 3$ method have rich biological associations, they may be drug target, they also may be pathogenic gene encodes annotated in OMIM and GWAS.

In order to further explicitly evaluate the biological functions of the $\mathrm{C} 3$ proteins, we used ClueGO [34] to conduct GO term (BP) enrichment analysis of these C3 proteins ( $p$ value $<0.01$ ) of these $\mathrm{C} 3$ proteins (Fig. 5). And enriched GO terms are indispensable for the human, such as: development of endocrine system, development of cardiomyocytes, transport of glucose across membranes, and positive regulation of collagen metabolism. Many of them are also associated with BRCA, which again indicates that it is feasible to apply C3 in multi-omics data and our method can be used to identify different biologically active $\mathrm{C} 3$ proteins from different omics.

\section{Discussion}

Disease module helps to understand the molecular mechanisms underlying diseases and identify novel drug targets. Topological properties of disease module in the human interactome are the basis for all network-based prioritization tools for studying human diseases. However, DAPs appear to form plenty of SCCs in the incomplete interactome. The problem we strive to solve is about how to determine their connectivity pattern and detect a concise disease module. To solve it, we proposed a C3 method to connect SCCs in the interactome by importing $\mathrm{C} 3$ proteins and $\mathrm{C} 3$ interactions with high connectivity. Our results highlight the role of $\mathrm{C} 3$ proteins and $\mathrm{C} 3$ interactions in connecting DAPs, which are dispersed as SCCs in incomplete interactome. Then we compared our method with DIAMOnD and random simulations to verify the succinctness and connectivity significance of the $\mathrm{C} 3$ module we obtained. We also got some unexpected results: the overlap between different omics data of the same disease is not high, which shows a clear omics specificity. From the perspective of biology, being the basis of disease characteristics, C3 proteins and C3 interactions may offer better and more accurate candidates. In the end, we hope that our method can help to study potential disease mechanisms, disease heterogeneity, drug response, capture novel pathways and genes.

\section{Conclusions}

In this work, we develop C3 method, which is proved to be able to effectively construct succinct disease modules with relatively few C3 proteins for 299 diseases from OMIM and GWAS databases and multi-omics data of BRCA from TCGA. And the disease module helps us to understand the disease pathways and identify pathogenic genes.

\section{Methods}

\section{Networks information}

For the sake of accuracy of our experiment, we used the human interactome, which is also used in DIAMOnD [9]: It derives from seven sources of protein interactions by Ghiassian SD et al.: (1) regulatory interactions derived from transcription factors binding 
a

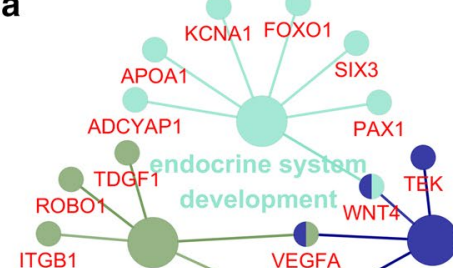

cell migration involved posittre regulation of in sprouting focal adhesion angiogenesis $\mathrm{ABL1}$ assembly

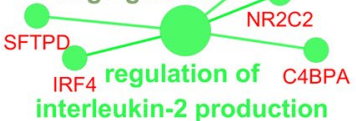

interleukin-2 production

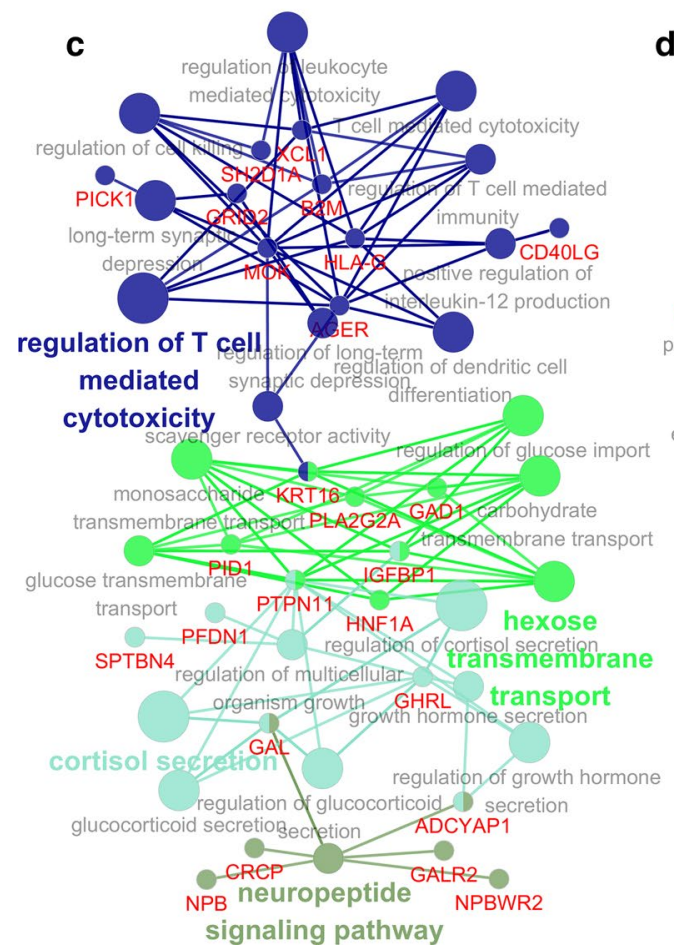

b

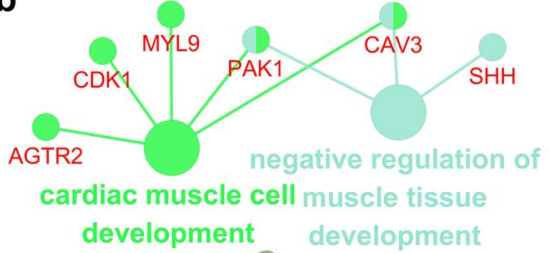

development

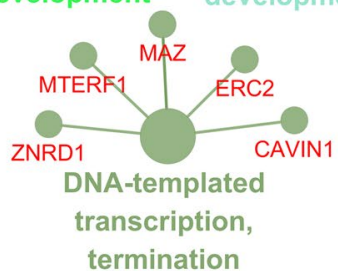

d

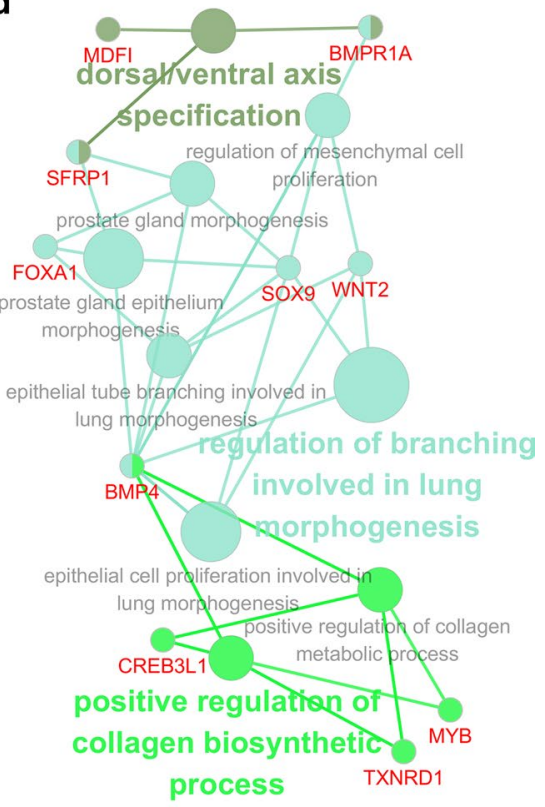

Fig. 5 Biological significance of C3 proteins from four omics data of BRCA. GO term (BP) enrichment analysis for C3 proteins obtained from a mRNA expression, $\mathbf{b}$ mutation, $\mathbf{c}$ DNA methylation, and $\mathbf{d}$ copy number variation. We used tool ClueGO to get the functional-gene network of the results. GO terms were statistically significant enriched for a set of genes, names of which are highlighted in red, other different colors represent different GO term clustering and the color of gene nodes represents the associated GO term. Only the most prominent term name in each group is colored and the larger term nodes are, the higher the enrichment degree is

to regulatory elements; (2) binary interactions from several yeast-two-hybrid highthroughput data sets and literature-curated data sets; (3) literature-curated interactions derived mostly from low-throughput experiments; (4) metabolic enzyme-coupled interactions; (5) protein complexes; (6) kinase-substrate pairs; and (7) signaling interactions. The union of all interactions from (1) to (7) yielded a network of 13,460 proteins that were interconnected by 141,296 interactions [10]. In addition, we used three other networks to supplement the experiment: We took a current interactome from BioGRID 
(https://thebiogrid.org/) and used the latest release compiled on June, 2019 and only selected human interactions, finally we got 17,741 genes and 338,793 interactions. And in order to avoid getting the observed performance which is solely due to study biases in human interactome, we got networks from Bioplex (https://bioplex.hms.harvard.edu/) and HuRI (https://interactome.dfci.harvard.edu/H_sapiens/), finally Bioplex had 10,961 genes and 56,553 interactions and HuRI had 7352 genes and 38,414 interactions. Network data was included into Additional file 1.

\section{DAPs of 299 diseases}

Menche et al. [10] integrated DAPs from OMIM (Online Mendelian Inheritance in Man; https://www.ncbi.nlm.nih.gov/omim) [22] which provides a link between genes and disease, and GWAS (Genome-Wide Association Study). The DAPs from GWAS were obtained from the Phenotype-Genotype Integrator database (PheGenI; www.ncbi. nlm.nih.gov/gap/PheGenI) [8] which merges GWAS catalog data with several databases housed at the NCBI (National Center for Biotechnology Information), and it supports searching annotated tables of genes based on phenotype. They used a genome-wide significance cutoff of $p$ value $\leq 5.0 \mathrm{e}-8$. Then in order to combine OMIM and GWAS they used the MeSH (Medical Subject Headings; https://www.nlm.nih.gov/mesh/) vocabulary. Using the hierarchical structure of the MeSH classification, in the result they found at least 20 DAPs and DAPs for which they have interaction information, and obtained 299 diseases and 3173 associated proteins.

\section{TCGA multi-omics data}

We obtained publicly available The Cancer Genome Atlas (TCGA) multi-omics data of BRCA from UCSC Cancer Browser [35] (https://xena.ucsc.edu). For mRNA expression, we used an R packet edge [36] and took the threshold of $|\log (\mathrm{FC})|>2$ and adj. $p$ value $<0.001$ to get 860 mRNA expression genes, where 632 mapped to human interactome were taken as DAPs. We used TCGA BRCA somatic mutation data and chose 889 genes that mutation counts $>10$ and mutation frequency $>0.01$, where 689 mapped were taken as DAPs. DNA methylation values, described as beta values, we used an $\mathrm{R}$ packet limma [37] and choose 837 genes of the threshold $|\log (\mathrm{FC})|>0.19$ and adj. $p$ value $<1.0 \mathrm{e}-14$, and only 367 mapped were taken as DAPs. BRCA thresholded genelevel copy number variation (CNV) estimated values to $-2,-1,0,1,2$, representing homozygous deletion, single copy deletion, diploid normal copy, low-level copy number amplification, or high-level copy number amplification. Then we obtained 863 genes of threshold values -2 and 2 and only 446 mapped were taken as DAPs. After processing the data, we obtained four altered data sets of multi-omics DAPs for BRCA, which are mRNA expression, mutation, DNA methylation and copy number variation.

\section{Biological validation data sets}

Following data is provided in Additional file 1.

(i) Gene expression of asthma specific compiled from eight sources: we selected eight expression data of direct asthma relevance from the Gene Expression Omnibus (GEO; https://www.ncbi.nlm.nih.gov/geo). We analyzed samples from GSE470, GSE473, GSE3004, GSE16032, GSE18965, GSE31773, GSE4302, GSE2125 with 
GEO2R, a total of 4111 differentially expressed genes were obtained where only 3273 in human interactome.

(ii) Gene ontology term similarity (BP): To elucidate the biological processes associated with the DAPs, we used the GO biological process category. We calculated the GO term similarity (BP) of all genes, took the top 4000 proteins with high similarity to all DAPs, and only 3154 in human interactome. The calculation tool was $\mathrm{R}$ package GOSemSim [26]

(iii) Disgenet: Disgenet data of asthma got from DisGeNET database (https://www. disgenet.org/). Sources of data include literature data, inferred data, and animal models data, totally we got 1817 genes associate with the asthma.

(iv) OMIM: OMIM genes got from Online Mendelian Inheritance in Man (OMIM; https://omim.org), totally we got 9915 genes.

(v) GWAS: GWAS genes got from Genome-Wide Association Studies (GWAS; https ://www.ebi.ac.uk/gwas), totally we got 15,858 genes.

(vi) Drug-target: Drug-target genes obtained from The Drug Gene Interaction Database (DGIdb; https://www.dgidb.org), totally we got 2993 genes

\section{C3 algorithm}

A basic detection process of C3 method is as follows: For a specific disease, we mapped its DAPs (seeds set $D$ ) into the network $G(N, E)$, where $N$ is the proteins set and $E$ is the interaction set. $N D$ is the set of non-seeds, $n_{0}=|N D|$ and $|\bullet|$ is the size of the corresponding set $[38,39]$.

\section{Export SCCs (ExScc):}

(1) Input:

Network $G(N, E)$;

Set $D$ of seeds;

(2) Preprocess:

Identify subgraph $D G$ of $D$ in $G(N, E)$ using networkx.Graph.subgraph

method[38]: $D G=$ G.subgraph $(D)$;

Identify SCCs of DG using networkx.connected_components method[39]:

$\operatorname{ExScc}(D)=$ networkx.connected_components $(D G)$;

(3) Output:

The set of SCCs, $s_{0}=|\operatorname{ExScc}(D)|$.

Then we considered the immediate neighbors of $D$ as the candidate proteins, and the adjacent edges of $D$ as the candidate interactions.

\section{Connectivity significance:}

For a candidate protein $i$, its connectivity degree to SCCs $k_{s}$ is defined as: 


$$
k_{s}=\mid(s \mid s \in S, \exists \text { a node } l \text { of } s,(i, l) \in E) \mid
$$

and its connectivity degree to non-seeds $k_{n d}$ is defined as:

$$
k_{n d}=|(j \mid j \neq i, j \in N D,(i, j) \in E)|
$$

Overall, the connectivity degree of $i$ is calculated as $k=k_{s}+k_{n d}$.

Analogously, for a candidate interaction $e(p, q)$ between protein $p$ and $q$, we quantified the $k_{s}$ and $k_{n d}$ for $e$ as:

$$
\begin{aligned}
& k_{s}=\mid(s \mid s \in S, \exists \text { a node } l \text { of } s,(p, l) \in E \text { or }(q, l) \in E) \mid \\
& k_{n d}=\mid(j \mid j \neq p, j \neq q, j \in N D,(p, j) \in E \text { or }(q, j) \in E) \mid
\end{aligned}
$$

The connectivity degree of $e$ is also calculated as $k=k_{s}+k_{n d}$.

Then, for randomly scattered SCCs, the connection probability that a candidate protein or a candidate interaction with a total connectivity degree $k$ has exactly $k_{s}$ links to SCCs is given by the hypergeometric [40]:

$$
p\left(k, k_{s}\right)=\frac{\left(\begin{array}{c}
s_{0} \\
k_{s}
\end{array}\right)\left(\begin{array}{c}
n_{0} \\
k_{n d}
\end{array}\right)}{\left(\begin{array}{c}
s_{0}+n_{0} \\
k
\end{array}\right)}
$$

To evaluate whether a candidate protein or a candidate interaction has more connections to SCCs than expected under null hypothesis, we calculate the connectivity significance $p$ value, i.e. the cumulative probability for the observed or any higher connectivity degree to SCCs:

$$
p \text { value }\left(k, k_{s}\right)=\sum_{k_{i}=k_{s}}^{k} p\left(k, k_{i}\right)
$$

This $p$ value is used as a connectivity significance measure, and is used to quantify the abilities of a candidate protein and a candidate interaction in connecting SCCs.

\section{$\mathrm{C} 3$ protein and $\mathrm{C} 3$ interaction:}

Then, we got the $\mathrm{C} 3$ disease module through a greedy process. In each iteration, $p$ value $<0.05$ and the lowest protein is selected as the C3 protein to connect SCCs and be added to $D$, then update $\operatorname{ExScc}(\mathrm{D})$. When $s_{0}$ is unchanged, $p$ value $<0.05$ and the lowest interaction is selected as the C3 interaction to connect SCCs and two proteins that occupy the endpoints of it, both are added to $D$, then update $\operatorname{ExScc}(D)$. Repeated these steps, when $s_{0}$ is unchanged, i.e. there are no $\mathrm{C} 3$ protein and $\mathrm{C} 3$ interaction that can connect SCCs with $p$ value $<0.05$, the process stopped, we obtained the C3 disease module.

The $\mathrm{C} 3$ proteins and the pair of proteins occupying the endpoints of $\mathrm{C} 3$ interactions are defined as intermediate proteins, which are collectively referred to as " $\mathrm{C} 3$ proteins" in the functional analysis. 


\section{Pseudocode:}

(1) Input:

Network $G(N, E)$;

Set $D$ of seeds;

(2) Loop:

Identify the set of SCCs of $D$ in $G(N, E), s_{0}=|\operatorname{ExScc}(D)|$;

Collect the immediate neighbor proteins of $D$ as candidate proteins into set C_P ;

Collect the candidate proteins interactions (don't include interactions with

$D)$ as candidate interactions into set $C_{-} I$;

$\forall i \in C_{-} P$, calculate connectivity significant $\mathrm{p}$-value of $i$;

Rank proteins according to $\mathrm{p}$-values;

If the top-ranked protein $i$ with $\mathrm{p}$-value $<0.05$ :

$i$ is a C3 protein, and $D=D \cup\{i\}$;

Update set $S$ of SCCs of $D$ in $G(N, E), s_{0}^{\prime}=|\operatorname{ExScc}(D)|$;

If $s_{0}^{\prime}=s_{0}$ :

$\forall e(p, q) \in C_{-} I$, Calculate connectivity significant $\mathrm{p}$-value of $e_{\text {; }}$

Rank interactions according to $\mathrm{p}$-values;

If the top-ranked interaction $e(p, q)$ with $p$-value $<0.05$ :

$e(p, q)$ is a C3 interaction, and $D=D \cup\{p, q\}$;

Update set $S$ of SCCs of $D$ in $G(N, E), s_{0}^{\prime \prime}=|\operatorname{ExScc}(D)|$;

If $s_{0}^{\prime \prime}=s_{0}$ :

Break;

(3) Output:

The largest connected component of $D$ in $G(N, E)$;

The largest connected component of $D$ in $G(N, E)$ is the disease module. Its time complexity is $O\left(n^{2}\right)$. We implemented C3 with Python and the codes can be downloaded from https://github.com/wangbingbo2019/C3.

\section{Randomized experiments}

For asthma, we conducted two randomized experiments. One is completely random, namely when we randomly selected 100 proteins from the network (the number consistent with DIAMOnD) using numpy.random.choice method [41] and added them to seed set $D$, we treated the largest connected component of $D$ as the random module. 
After 1000 random times, we got the mean $\mu$ and standard deviation $\sigma$ of the number of connected seeds in random modules. Z-score of true (C3 or DIAMOnD) disease module with $T$ (\#DPcC or \#DPcD) connected seeds is given by the following:

$$
z \text { score }=\frac{T-\mu}{\sigma}
$$

The other is that instead of randomly selecting from the network, we randomly selected 100 proteins from the immediate neighbors of $D$ and added them to seed set $D$ to get the final random module.After 1000 random times, $\mathrm{z}$-score is also calculated by Eq. (7).

For BRCA, we randomly selected 40 random proteins matching size and degree distribution as BRCA seeds using numpy.random.choice method [41] for random experiments.After 1000 random times, we got the mean and standard deviation of the number of $\mathrm{cP}$ and $\mathrm{iP}$ in random modules. Z-score of $\mathrm{C} 3$ disease module with true number of $\mathrm{cP}$ and iP is also given by Eq. (7).

\section{Supplementary information}

Supplementary information accompanies this paper at https://doi.org/10.1186/s12859-020-03769-y.

Additional file 1: Supplementary data and results.

\section{Abbreviations}

C3: Connect separate connected components; SCCs: Separate connected components; DAPs: Disease-associated proteins; OMIM: Online Mendelian Inheritance in Man; GWAS: Genome-Wide Association Study; LCC: Largest connected component; TCGA: The Cancer Genome Atlas; BRCA: BReast CAncer; DPCD: Disease proteins contained in DIAMOnD module; DPCC: Disease proteins contained in C3 module; CP: Connected proteins; iP: Intermediate proteins.

\section{Acknowledgements}

The authors would like to thank all the editors and anonymous reviewers for their constructive advice.

\section{Authors' contributions}

BBW, JH: conceived and designed the experiments. JH performed the experiments. CXZ, YJZ, LY, XLG, LG, YRC: analyzed the data. BBW, JH, CXZ, YJW, YRC: wrote the paper. Bingbo Wang and Jie Hu contributed equally to this work. All authors read and approved the final manuscript.

\section{Funding}

This project has been supported by the National Natural Science Foundation of China (Nos. 61772395, 61532014, 61672406, 61672407, 61702396 and 61702397), China Postdoctoral Science Foundation (No. 2015M582620), Fundamental Research Funds for the Central Universities (No. JB190306) and Shanghai Municipal Science and Technology Major Project (No. 2018SHZDZX01), LCNBI and ZJLab for the financial support, some websites for data online. The funding bodies had no role in the design of the study; collection, analysis, and interpretation of data; or in writing the manuscript.

\section{Availability of data and materials}

All supporting files can be downloaded from https://github.com/wangbingbo2019/C3 including: the networks data, disease genes from OMIM and GWAS, some data sets for validation, the C3 tool in Python, and the results of C3 modules.

\section{Ethics approval and consent to participate}

Not applicable.

\section{Consent for publication}

Not applicable.

\section{Competing interests}

The authors declare that they have no competing interests.

\section{Author details}

${ }^{1}$ School of Computer Science and Technology, Xidian University, Xi'an, People's Republic of China. ${ }^{2}$ School of Humanities and Foreign Languages, Xi'an University of Technology, Xi'an, People's Republic of China. ${ }^{3}$ The First Affiliated Hospital of Xi'an Jiaotong University, Xi'an, People's Republic of China.

Received: 20 February 2020 Accepted: 20 September 2020

Published online: 02 October 2020 


\section{References}

1. Goh K-I, Cusick ME, Valle D, Childs B, Vidal M, Barabási A-L. The human disease network. Proc Natl Acad Sci. 2007;104:8685-90.

2. Zanzoni A, Soler-López M, Aloy P. A network medicine approach to human disease. FEBS Lett. 2009;583:1759-65.

3. Barabási A-L, Gulbahce N, Loscalzo J. Network medicine: a network-based approach to human disease. Nat Rev Genet. 2011;12:56-68.

4. Schadt EE. Molecular networks as sensors and drivers of common human diseases. Nature. 2009:461:218-23.

5. Pawson T, Linding R. Network medicine. FEBS Lett. 2008;582:1266-70.

6. Califano A, Butte AJ, Friend S, Ideker T, Schadt E. Leveraging models of cell regulation and GWAS data in integrative network-based association studies. Nat Genet. 2012;44:841-7.

7. Venkatesan K, Rual J-F, Vazquez A, StelzI U, Lemmens I, Hirozane-Kishikawa T, et al. An empirical framework for binary interactome mapping. Nat Methods. 2009;6:83-90.

8. Ramos EM, Hoffman D, Junkins HA, Maglott D, Phan L, Sherry ST, et al. Phenotype-genotype integrator (PheGenl): synthesizing genome-wide association study (GWAS) data with existing genomic resources. Eur J Hum Genet. 2014;22:144-7.

9. Ghiassian SD, Menche J, Barabási A-L. A DIseAse MOdule Detection (DIAMOnD) algorithm derived from a systematic analysis of connectivity patterns of disease proteins in the human interactome. PLOS Comput Biol. 2015:11:e1004120.

10. Menche J, Sharma A, Kitsak M, Ghiassian SD, Vidal M, Loscalzo J, et al. Uncovering disease-disease relationships through the incomplete interactome. Science. 2015;347:1257601.

11. Agrawal M, Zitnik M, Leskovec J. Large-scale analysis of disease pathways in the human interactome. Pac Symp Biocomput Pac Symp Biocomput. 2018:23:111-22.

12. Vinayagam A, Gibson TE, Lee H-J, Yilmazel B, Roesel C, Hu Y, et al. Controllability analysis of the directed human protein interaction network identifies disease genes and drug targets. Proc Natl Acad Sci U S A. 2016;113:4976-81.

13. Ghiassian SD, Menche J, Chasman DI, Giulianini F, Wang R, Ricchiuto P, et al. Endophenotype network models: common core of complex diseases. Sci Rep. 2016;6:27414.

14. Huttlin EL, Bruckner RJ, Paulo JA, Cannon JR, Ting L, Baltier K, et al. Architecture of the human interactome defines protein communities and disease networks. Nature. 2017;545:505-9.

15. Liu C-C, Tseng Y-T, Li W, Wu C-Y, Mayzus I, Rzhetsky A, et al. DiseaseConnect: a comprehensive web server for mechanism-based disease-disease connections. Nucleic Acids Res. 2014;42(Web Server issue):W137-46.

16. Boyle EA, Li YI, Pritchard JK. An expanded view of complex traits: from polygenic to omnigenic. Cell. 2017;169:1177-86.

17. Wray NR, Wijmenga C, Sullivan PF, Yang J, Visscher PM. Common disease is more complex than implied by the core gene omnigenic model. Cell. 2018;173:1573-80.

18. Sharma A, Menche J, Huang CC, Ort T, Zhou X, Kitsak M, et al. A disease module in the interactome explains disease heterogeneity, drug response and captures novel pathways and genes in asthma. Hum Mol Genet. 2015;24:3005-20.

19. Agrawal M, Zitnik M, Leskovec J. Large-scale analysis of disease pathways in the human interactome. bioRxiv 189787; 2017.

20. Navlakha S, Kingsford C. The power of protein interaction networks for associating genes with diseases. Bioinformatics. 2010;26:1057-63.

21. Radicchi F, Castellano C, Cecconi F, Loreto V, Parisi D. Defining and identifying communities in networks. Proc Natl Acad Sci. 2004;101:2658-63.

22. Hamosh A, Scott AF, Amberger JS, Bocchini CA, McKusick VA. Online Mendelian Inheritance in Man (OMIM), a knowledgebase of human genes and genetic disorders. Nucleic Acids Res. 2005;33(Database Issue):D514-7.

23. Sadeghi A, Fröhlich $H$. Steiner tree methods for optimal sub-network identification: an empirical study. BMC Bioinform. 2013;14:144

24. Sanchez-Vega F, Mina M, Armenia J, Chatila WK, Luna A, La KC, et al. Oncogenic signaling pathways in the cancer genome atlas. Cell. 2018;173(321-337):e10

25. Overview of NetworkX-NetworkX 2.4 documentation. https://networkx.github.io/documentation/stable/ Accessed 15 July 2020.

26. Yu G, Li F, Qin Y, Bo X, Wu Y, Wang S. GOSemSim: an R package for measuring semantic similarity among GO terms and gene products. Bioinforma Oxf Engl. 2010;26:976-8.

27. Huang DW, Sherman BT, Tan Q, Kir J, Liu D, Bryant D, et al. DAVID Bioinformatics Resources: expanded annotation database and novel algorithms to better extract biology from large gene lists. Nucleic Acids Res. 2007;35(Web Server issue):W169-75.

28. LuY, Ho R, Lim TK, Kuan WS, Goh DYT, Mahadevan M, et al. Neuropeptide Y may mediate psychological stress and enhance TH2 inflammatory response in asthma. J Allergy Clin Immunol. 2015;135(1061-1063):e4.

29. Izuhara K, Arima K. Signal transduction of IL-13 and its role in the pathogenesis of bronchial asthma. Drug News Perspect. 2004;17:91-8.

30. Doorley LA, LeMessurier KS, Iverson AR, Palipane M, Samarasinghe AE. Humoral immune responses during asthma and influenza co-morbidity in mice. Immunobiology. 2017:222:1064-73.

31. Rutkowski R, Moniuszko T, Stasiak-Barmuta A, Kosztyła-Hojna B, Alifier M, Rutkowski K, et al. CD80 and CD86 expression on LPS-stimulated monocytes and the effect of CD80 and CD86 blockade on IL-4 and IFN-gamma production in nonatopic bronchial asthma. Arch Immunol Ther Exp (Warsz). 2003;51:421-8.

32. Pistiner M, Hunninghake GM, Soto-Quiros ME, Avila L, Murphy A, Lasky-Su J, et al. Polymorphisms in IL12A and cockroach allergy in children with asthma. Clin Mol Allergy CMA. 2008;6:6.

33. Liu Y, Saccucci P, Qi H, Wu HC, Zhao F, Dai Y, et al. ADA polymorphisms and asthma: a study in the Chinese Han population. J Asthma Off J Assoc Care Asthma. 2006;43:203-6.

34. Bindea G, Mlecnik B, Hackl H, Charoentong P, Tosolini M, Kirilovsky A, et al. ClueGO: a Cytoscape plug-in to decipher functionally grouped gene ontology and pathway annotation networks. Bioinforma Oxf Engl. 2009;25:1091-3. 
35. M Goldman, B Craft, M Hastie, K Repečka, A Kamath, F McDade, D Rogers, AN Brooks, J Zhu, D Haussler. The UCSC Xena Platform for cancer genomics data visualization and interpretation. bioRxiv. https://doi.org/10.1101/326470v4. Accessed 3 Apr 2019.

36. Leek JT, Monsen E, Dabney AR, Storey JD. EDGE: extraction and analysis of differential gene expression. Bioinforma Oxf Engl. 2006;22:507-8

37. Ritchie ME, Phipson B, Wu D, Hu Y, Law CW, Shi W, et al. limma powers differential expression analyses for RNAsequencing and microarray studies. Nucleic Acids Res. 2015;43:e47.

38. networkx.Graph.subgraph—NetworkX 2.4 documentation. https://networkx.github.io/documentation/stable/refer ence/classes/generated/networkx.Graph.subgraph.html. Accessed 27 June 2020

39. Components-NetworkX 2.4 documentation. https://networkx.github.io/documentation/stable/reference/algor ithms/component.html. Accessed 27 June 2020.

40. John AR. Mathematical statistics and data analysis. 3rd ed. Pacific Grove: Duxbury Press; 2007.

41. numpy.random.choice-NumPy v1.13 manual. https://docs.scipy.org/doc/numpy-1.13.0/reference/generated/ numpy.random.choice.html. Accessed 28 June 2020.

\section{Publisher's Note}

Springer Nature remains neutral with regard to jurisdictional claims in published maps and institutional affiliations.

- fast, convenient online submission

- thorough peer review by experienced researchers in your field

- rapid publication on acceptance

- support for research data, including large and complex data types

- gold Open Access which fosters wider collaboration and increased citations

- maximum visibility for your research: over 100M website views per year

At BMC, research is always in progress.

Learn more biomedcentral.com/submissions 